\title{
2,5-Dimethyl-3,6-bis[(2,6-diisopropylphenylimino)methyl]- and 2,6-Bis[(2,6-diisopropylphenylimino)methyl]pyrazine: Two New Chelating Ligands for Transition Metal Complexes
}

\author{
Herbert Schumann and He-Kuan Luo \\ Institut für Chemie, Technische Universität Berlin, Straße des 17. Juni 135, D-10623 Berlin, \\ Germany
}

Reprint requests to H. Schumann. E-mail: Schumann@chem.tu-berlin.de

Z. Naturforsch. 60b, 22-24 (2005); received August 26, 2004

\begin{abstract}
The reaction of tetramethylpyrazine with $\mathrm{SeO}_{2}$ yields 2,5-dimethylpyrazine-3,6-dicarboxaldehyde (1) from which the 2,5-dimethyl-3,6-bis[(2,6-diisopropylphenylimino)methyl]pyrazine (2) was synthesized by treatment with 2 equivalents of 2,6-diisopropylaniline. 2,6-Dimethylpyrazine reacts with benzaldehyde to give 2,6-distyrylpyrazine (3). Ozonolysis of $\mathbf{3}$, followed by treatment with $\mathrm{Na}_{2} \mathrm{SO}_{3}$ and 2,6-diisopropylaniline resulted in the formation of 2,6-bis[(2,6-diisopropylphenylimino)methyl]pyrazine (5) together with [(2,6-diisopropylphenylimino)methyl]benzene (6).
\end{abstract}

Key words: Diimine, Dialdehyde, Pyrazine, Chelating Ligands

\section{Introduction}

Nitrogen containing organic compounds, in particular pyridine and/or imine derivatives, are very important ligands widely used in various organometallic complexes for olefin oligomerization and polymerization [1]. In general, the performance of the catalysts is regulated by the structure of the ligands. Therefore the synthesis of new ligands is one of the keys for the development of new catalysts.

Usually, imine containing ligands are prepared via condensation of carbonyl compounds with aniline derivatives bearing bulky substituents [1]. Because of the smoothly proceeding condensation of the carbonyl group with aniline under mild conditions [2], the key to the preparation of such ligands is the synthesis of starting materials containing suitable carbonyl groups.

Widely used methods to prepare aldehydes are the oxidation of $\alpha$-methyl groups or of unsaturated compounds by $\mathrm{SeO}_{2}$ [3], $\mathrm{O}_{3}$ [4], and other oxidants [5]. In this paper we describe the synthesis of two new chelating ligand systems derived from 2,5dimethylpyrazine-3,6-dicarboxaldehyde and pyrazine3,6-dicarboxaldehyde.

\section{Results and Discussion}

The reaction of 2,3,5,6-tetramethylpyrazine with a tenfold amount of $\mathrm{SeO}_{2}$ in wet dioxane results in the formation of 2,5-dimethylpyrazine-3,6-dicarboxaldehyde (1) in $26 \%$ yield as a yellow powder. This product reacts with 2 equivalents of 2,6diisopropylaniline yielding yellow 2,5-dimethyl-3,6bis[(2,6-diisopropylphenylimino)methyl]pyrazine (2) in $70 \%$ yield (Scheme 1 ).

First attempts to prepare 2,6-bis[(2,6-diisopropylphenylimino)methyl]pyrazine in analogy to 2 via $\mathrm{SeO}_{2}$ oxidation of 2,6-dimethylpyrazine failed. The reaction temperature of more than $100{ }^{\circ} \mathrm{C}$ does not allow the isolation of pyrazine-2,6-dicarboxaldehyde, which decomposes quickly after generation. Therefore we chose another route which can be used for the synthesis of thermally and oxidatively labile aldehydes, vic. the oxidation of olefins with ozone, the reduction of the resulting ozonides with $\mathrm{Na}_{2} \mathrm{SO}_{3}$, followed by condensation with 2,6-diisopropylaniline [4].

Using a modification of the method successfully employed for the synthesis of 2,5-distyrylpyrazine [4b, 6], 2,6-distyrylpyrazine (3) was prepared in high yield by condensation of 2,6-dimethylpyrazine with benzaldehyde (Scheme 2).

Treatment of $\mathbf{3}$ with ozone at $-50{ }^{\circ} \mathrm{C}$, followed by reduction of the ozonide formed with $\mathrm{Na}_{2} \mathrm{SO}_{3}$ in methanol at $-50{ }^{\circ} \mathrm{C}$ and condensation of the dialdehyde 4 with 2,6-diisopropylaniline at room temperature resulted in 2,6-bis[(2,6-diisopropylphenylimino)methyl]pyrazine (5), and [(2,6-diisopropylphen- 


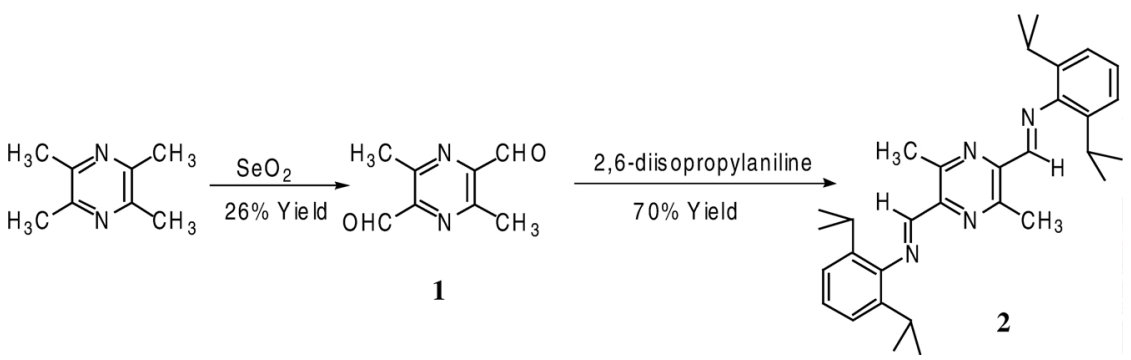<smiles>Cc1cncc(C)n1</smiles>

Scheme 2.

ylimino)methyl]benzene (6) which were separated by column chromatography (Scheme 3). The diozonide as well as the dialdehyde $\mathbf{4}$ were not isolated and characterized, but used in the "one-pot" reaction without further purification.

In conclusion, two new poly- $N$-functional Schiffbase ligands were synthesized via the dialdehydes. Each of them has two imine groups and two nitrogen atoms in the aromatic pyrazine system. Obviously, both are very good potential ligands, of which $\mathbf{2}$ can be used as a bis-chelating ligand which can coordinate with two metal centers, and $\mathbf{5}$ as a tridendate ligand to prepare various transition metal complexes for catalysis.

\section{Experimental Section}

All operations were carried out in an atmosphere of dry, oxygen-free nitrogen using standard Schlenk techniques. Solvents were dried over sodium, purified and saturated with nitrogen prior to use. Elemental analyses were performed on a Perkin-Elmer 240C elemental analyzer. Mass spectra $(\mathrm{EI}, 70 \mathrm{eV}$ ) were obtained using a Varian MAT $311 \mathrm{~A}$ instrument. ${ }^{1} \mathrm{H}$ and ${ }^{13} \mathrm{C}$ NMR spectra were recorded on a Bruker ARX 200 (200 and $50.32 \mathrm{MHz}$, respectively) and a Bruker ARX 400 (400 and 100.64 MHz, respectively) spectrometer in $\mathrm{CDCl}_{3}$ unless otherwise stated.

2,5-Dimethylpyrazine-3,6-dicarboxaldehyde (1): Tetramethylpyrazine $(1.9 \mathrm{~g}, 14 \mathrm{mmol})$ and $\mathrm{SeO}_{2}(15.4 \mathrm{~g}$, $140 \mathrm{mmol})$ were dissolved in a mixture of dioxane $(240 \mathrm{ml})$ and water $(10 \mathrm{ml})$ and refluxed for $5 \mathrm{~h}$. After filtration of the black powder received, the clear filtrate was concentrated nearly to dryness. Toluene $(120 \mathrm{ml})$ was added, the mixture was refluxed for $30 \mathrm{~min}$, decanted from the remaining residue and the solvents were evaporated to leave a volume of $10 \mathrm{ml}$. Yellow needles of $\mathbf{1}$ separated and were collected by filtra-
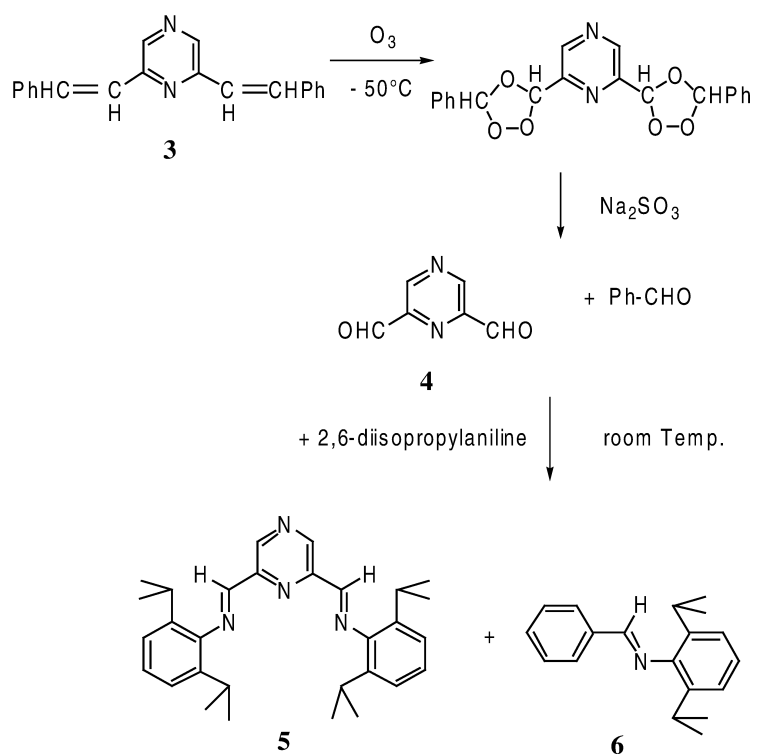

Scheme 3.

tion. Yield: $0,60 \mathrm{~g}(26 \%)$. M.p. $140-142{ }^{\circ} \mathrm{C} .-{ }^{1} \mathrm{H}$ NMR (200 MHz): $\delta=2.86$ (s, $\left.6 \mathrm{H}, \mathrm{CH}_{3}\right), 10.16$ (s, $\left.2 \mathrm{H}, \mathrm{CHO}\right)$. ${ }^{13} \mathrm{C}$ NMR (50.32 MHz): $\delta=21.36\left(\mathrm{CH}_{3}\right), 144.65\left(\mathrm{CCH}_{3}\right)$, 151.95 (CCHO), 193.56 (CCHO).

2,5-Dimethyl-3,6-bis[(2,6-diisopropylphenylmino)me-

thyl]pyrazine (2): A mixture of $1(0,58 \mathrm{~g}, 3.5 \mathrm{mmol})$, 2,6-diisopropylaniline $(1,4 \mathrm{ml})$ and acetic acid $(0,1 \mathrm{ml})$ in ethanol $(8 \mathrm{ml})$ was refluxed for $15 \mathrm{~h}$ with stirring. Filtration of the reaction mixture at room temperature and washing of the resulting yellow powder with ethanol $(5 \mathrm{ml})$ yielded 2 (1.19 g, 70\%). M.p. $220-222{ }^{\circ} \mathrm{C} .-{ }^{1} \mathrm{H}$ NMR $(200 \mathrm{MHz})$ : $\delta=1.26\left[\mathrm{~d},{ }^{3} J=6.9 \mathrm{~Hz}, 24 \mathrm{H}, \mathrm{CH}\left(\mathrm{CH}_{3}\right)_{2}\right.$ ], 3.08 [sept, $\left.{ }^{3} J=6.9 \mathrm{~Hz}, 4 \mathrm{H}, \mathrm{CH}\left(\mathrm{CH}_{3}\right)_{2}\right], 3.11$ [s, $\left.6 \mathrm{H}, \mathrm{CH}_{3}(\mathrm{pyr})\right]$, $7.21-7.27(\mathrm{~m}, 6 \mathrm{H}, \mathrm{Ph}), 8.57(\mathrm{~s}, 2 \mathrm{H}, \mathrm{CH}=\mathrm{N}) .-{ }^{13} \mathrm{C} \mathrm{NMR}$ (50.32 MHz): $\delta=23.24\left[\mathrm{CH}_{3}(\right.$ py) $], 23.45\left(\mathrm{CHCH}_{3}\right), 28.02$ $\left(\mathrm{CHCH}_{3}\right), 123.01(\mathrm{Ph}), 124.63(\mathrm{Ph}), 136.72(\mathrm{Ph}), 145.32$ (C-pyr), $149.02(\mathrm{Ph}), 151.24(\mathrm{C}-\mathrm{pyr}), 163.53(\mathrm{CH}=\mathrm{N})$. $\mathrm{C}_{32} \mathrm{H}_{42} \mathrm{~N}_{4}$ (482.71): calcd. C 79.62, H 8.77, N 11.61; found C 79.68, H 8.53, N 11.78.

2,6-Distyrylpyrazine (3): A mixture of 2,6-dimethylpyrazine $(2.1 \mathrm{~g}, 20 \mathrm{mmol}), \mathrm{ZnCl}_{2}(2.8 \mathrm{~g}, 20 \mathrm{mmol})$ and benzalde- 
hyde $(30 \mathrm{ml})$ was heated to $155^{\circ} \mathrm{C}$ for $8 \mathrm{~h}$ with stirring. Then ethanol $(30 \mathrm{ml})$ was added, the reaction mixture refluxed for $30 \mathrm{~min}$ and cooled to room temperature and filtered. The remaining yellow powder was washed with ethanol $(20 \mathrm{ml})$ and dried in vacuum yielding $5.45 \mathrm{~g}(100 \%)$ of 3. M.p. $229-$ $230{ }^{\circ} \mathrm{C}$.

2,6-Bis[(2,6-diisopropylphenylimino)methyl]pyrazine (5) and [(2,6-Diisopropylphenylimino)methyl]benzene (6): A solution of $3(2.1 \mathrm{~g}, 20 \mathrm{mmol})$ in methanol $(200 \mathrm{ml})$ was cooled in a $\mathrm{CO}_{2}$ /isopropanol bath to $-50{ }^{\circ} \mathrm{C}$ and $\mathrm{O}_{3}$ was bubbled through the stirred solution until complete dissoluation of $\mathbf{3}$ and appearance of the pale blue colour of dissolved $\mathrm{O}_{3}(6-8 \mathrm{~h})$. Excess of $\mathrm{O}_{3}$ was then displaced by bubbling $\mathrm{N}_{2}$ through the solution until the pale blue colour disappeared. Finely ground $\mathrm{Na}_{2} \mathrm{SO}_{3}(8.25 \mathrm{~g})$ was added and the solution was stirred for $5 \mathrm{~h}$ at $-50{ }^{\circ} \mathrm{C}$. The reaction mixture was warmed to room temperature and a white solid was filtered off. 2,6-Diisopropylaniline $(6,2 \mathrm{ml})$ and acetic acid $(0,3 \mathrm{ml})$ were added to the remaining clear solution, which was then stirred for one week at room temperature. Evaporation of methanol under vacuum left a few $\mathrm{ml}$ of a yellow solution, from which $0,5 \mathrm{~g}(16 \%)$ of 5 and $0,45 \mathrm{~g} \mathrm{(12 \% )}$ of $\mathbf{6}$ were isolated by column chromatography (toluene).
5: M.p. $133-134{ }^{\circ} \mathrm{C} .-{ }^{1} \mathrm{H}$ NMR (400 MHz): $\delta=1.26$ (d, ${ }^{3} J=6.9 \mathrm{~Hz}, 24 \mathrm{H}, \mathrm{CH}_{3}$ ), 3.04 [sept, ${ }^{3} J=6.9 \mathrm{~Hz}, 4 \mathrm{H}$, $\left.\mathrm{CH}\left(\mathrm{CH}_{3}\right)_{2}\right], 7.21-7.27(\mathrm{~m}, 6 \mathrm{H}, \mathrm{Ph}), 8.47(\mathrm{~s}, 2 \mathrm{H}, \mathrm{CH}=\mathrm{N})$, 9.65 [s, $2 \mathrm{H}, \mathrm{H}(\mathrm{pyr})] .-{ }^{13} \mathrm{C}$ NMR $(100,64 \mathrm{MHz}): \delta=23.39$ $\left(\mathrm{CH}_{3}\right), 27.98\left(\mathrm{CHCH}_{3}\right), 123.17(\mathrm{Ph}), 124.99(\mathrm{Ph}), 137.03$ (Ph), 144.25 (C-pyr), 147.94 (Ph), 148.49 (C-pyr), 160.97 $(\mathrm{CH}=\mathrm{N}) .-\mathrm{C}_{30} \mathrm{H}_{38} \mathrm{~N}_{4}$ (445.66): calcd. C 79.25, H 8.42, $\mathrm{N}$ 12.32; found C 79.09, H 8.58, N 12.31.

6: M.p. $123-124{ }^{\circ} \mathrm{C} .-{ }^{1} \mathrm{H}-\mathrm{NMR}(400 \mathrm{MHz}): \delta=1.24$ (d, $\left.{ }^{3} J=6.8 \mathrm{~Hz}, 12 \mathrm{H}, \mathrm{CH}_{3}\right), 3.06\left[\mathrm{sept},{ }^{3} \mathrm{~J}=6.8 \mathrm{~Hz}, 2 \mathrm{H}\right.$, $\mathrm{CH}\left(\mathrm{CH}_{3}\right)_{2}$ ], $7.18-7.24\left(\mathrm{~m}, 3 \mathrm{H}, \mathrm{Ph}_{\mathrm{m}, \mathrm{p}}\right), 7.98-8.00(\mathrm{~m}, 2 \mathrm{H}$, $\left.\mathrm{Ph}_{\mathrm{o}}\right), 8.27(\mathrm{~s}, 1 \mathrm{H}, \mathrm{CH}=\mathrm{N}) .-{ }^{13} \mathrm{C} \mathrm{NMR}(100,64 \mathrm{MHz}): \delta=$ $23.42\left(\mathrm{CH}_{3}\right), 27.86\left(\mathrm{CHCH}_{3}\right), 123.02\left(\mathrm{C}_{6} \mathrm{H}_{3}-3,5\right), 124.13$ $\left(\mathrm{C}_{6} \mathrm{H}_{3}-4\right), 128.59\left(\mathrm{C}_{6} \mathrm{H}_{5}-3,5\right), 128.83\left(\mathrm{C}_{6} \mathrm{H}_{5}-2,6\right), 131.44$ $\left(\mathrm{C}_{6} \mathrm{H}_{5}-4\right), 136.01 \quad\left(\mathrm{C}_{6} \mathrm{H}_{5}-1\right), 137.60\left(\mathrm{C}_{6} \mathrm{H}_{3}-2,6\right), 149.24$ $\left(\mathrm{C}_{6} \mathrm{H}_{3}-1\right), 162.03(\mathrm{CH}=\mathrm{N})$.

\section{Acknowledgements}

We thank the Alexander von Humboldt Foundation, the Deutsche Forschungsgemeinschaft (Graduiertenkolleg "Synthetische, mechanistische und reaktionstechnische Aspekte von Metallkatalysatoren") and the Fonds der Chemischen Industrie for support of this work.
[1] a) T. V. Laine, U. Piironen, K. Lappalainen, M. Klinga, E. Aitola, M. Lekelä, J. Organomet. Chem. 606, 112 (2000); b) S. A. Svejda, M. Brookhart, Organometallics 18, 65 (1999); c) V.C. Gibson, S. K. Spitzmesser, Chem. Rev. 103, 283 (2003); d) G. J.P. Britovsek, V. C. Gibson, Angew. Chem. 111, 448 (1999); Angew. Chem. Int. Ed. 38, 428 (1999); e) S.D. Ittel, L. K. Johnson, M. Brookhart, Chem. Rev. 100, 1169 (2000); f) B. L. Small, M. Brookhart, A. M. A. Bennett, J. Am. Chem. Soc. 120, 4049 (1998); g) A. M. A. Bennett, PCT International Patent WO 98/27124 (1998); h) G. J.P. Britovsek, V.C. Gibson, B.S. Kimberley, P.J. Maddox, S. J. McTavish, G. A. Solan, A. J.P. White, D. J. Williams, Chem. Commun. 849 (1998); i) G.J.P. Britovsek, B. A. Dorer, V. C. Gibson, B. S. Kimberley, WO 99/12981 (1999); j) M. A. Esteruelas, A. M. Lopez, L. Mendez, M. Olivan, E. Onate, Organometallics 22, 395 (2003); k) J. H. Browine, M. C. Baird, L. N. Zakharov, A.L. Rheingold, Organometallics 22, 33 (2003).

[2] a) H. van der Poel, G. van Koten, Synth. Commun. 8, 305 (1978); b) H. A. Zhong, J. A. Labinger, J.E. Bercaw, J. Am. Chem. Soc. 124, 1378 (2002).

[3] a) C. J. Chandler, L.W. Deady, J. A. Reiss, J. Heterocycl. Chem. 18, 599 (1981); b) L. Garuti, A. Ferranti, S. Burnelli, L. Varoli, G. Giovanninetti, P. Brigidi, A. Casolari, Boll. Chim. Farm. 128, 136 (1989); c) E. J.
Corey, J.P. Schaefer, J. Am. Chem. Soc. 82, 918 (1960); d) J. Francis, J. K. Landquist, A. A. Levi, J. A. Silk, J. M. Thorp, Biochem. J. 63, 455 (1956); e) E. J. Moriconi, A. J. Fritsch, J. Org. Chem. 30, 1542 (1965); f) A. G. Caldwell, J. Chem. Soc. 2035 (1952).

[4] a) S. Brooker, R. J. Kelly, J. Chem. Soc. Dalton Trans. 2117 (1996); b) R. H. Wiley, J. Macromol. Sci. Chem. A 24, 1184 (1987); c) A. Hirschberg, D. P. Smith, J. Heterocycl. Chem. 3, 103 (1966); d) J. J. Pappas, W.P. Keaveney, E. Gancher, M. Berger, Tetrahedron Lett. 36, 4273 (1966); e) D. Gupta, R. Soman, S. Dev, Tetrahedron 38, 3013 (1982); f) W. S. Knowles, Q.E. Thompson, J. Org. Chem. 25, 1031 (1960).

[5] a) S. V. Lieberman, R. Connor, Org. Syn., Coll. 2, 441 (1943); b) J. Thiele, E. Winter, Ann. 311, 356 (1900); c) T. Nishimura, Org. Syn., Coll. 4, 713 (1963); d) W. H. Hartford, M. Darrin, Chem. Rev. 58, 1 (1958); e) W. S. Trahanovsky, L. B. Young, J. Org. Chem. 31, 2033 (1966); f) R.P. Kreh, R. M. Spotnitz, J. T. Lundquist, J. Org. Chem. 54, 1526 (1989); g) D. H. R. Barton, R. A. H. F. Hui, S. V. Ley, J. Chem. Soc. Perkin Trans. 1, 2179 (1982); h) W. S. Li, L. K. Liu, Synthesis 293 (1989); i) L. Syper, Tetrahedron Lett. 4193 (1967).

[6] a) R. N. Castle, K. Kaji, J. Heterocycl. Chem. 2, 463 (1965); b) M. Hasegawa, Y. Suzuki, F. Suzuki, H. Nakanishi, J. Polym. Sci. Part A-1, 7, 743 (1969). 